\title{
Genetically encoded far-red fluorescent sensors for caspase-3 activity
}

\author{
Olga A. Zlobovskaya1, Tatiana F. Sergeeva², Marina V. Shirmanova², Varvara V. Dudenkova ${ }^{2,3}$, \\ George V. Sharonov4, Elena V. Zagaynova², and Konstantin A. Lukyanov ${ }^{1}$ \\ ${ }^{1}$ Shemyakin-Ovchinnikov Institute of Bioorganic Chemistry, Moscow, Russia, ${ }^{2}$ Nizhny Novgorod State \\ Medical Academy, Nizhny Novgorod, Russia, ${ }^{3}$ Nizhny Novgorod State University, Nizhny Novgorod, \\ Russia, and ${ }^{4}$ Faculty of Medicine, Lomonosov Moscow State University, Moscow, Russia
}

BioTechniques 60:62-68 (February 2016) doi 10.2144/000114377

Keywords: green fluorescent protein (GFP); bacteriophytochrome-based infrared fluorescent protein; genetically encoded fluorescent sensor; FRET; caspase-3; apoptosis

Caspase-3 is a key effector caspase that is activated in both extrinsic and intrinsic pathways of apoptosis. Available fluorescent sensors for caspase-3 activity operate in relatively short wavelength regions and are nonoptimal for multiparameter microscopy and whole-body imaging. In the present work, we developed new genetically encoded sensors for caspase-3 activity possessing the most red-shifted spectra to date. These consist of Förster resonance energy transfer (FRET) pairs in which a far-red fluorescent protein (mKate2 or eqFP650) is connected to the infrared fluorescent protein iRFP through a linker containing the DEVD caspase-3 cleavage site. During staurosporine-induced apoptosis of mammalian cells (HeLa and CT26), both mKate2-DEVD-iRFP and eqFP650-DEVD-iRFP sensors showed a robust response (1.6fold increase of the donor fluorescence intensity). However, eqFP650-DEVD-iRFP displayed aggregation in some cells. For stably transfected CT26 mKate2-DEVD-iRFP cells, fluorescence lifetime imaging (FLIM) enabled us to detect caspase-3 activation due to the increase of mKate2 donor fluorescence lifetime from 1.45 to 2.05 ns. We took advantage of the strongly red-shifted spectrum of mKate2-DEVD-iRFP to perform simultaneous imaging of EGFP-Bax translocation during apoptosis. We conclude that mKate2-DEVD-iRFP is well-suited for multiparameter imaging and also potentially beneficial for in vivo imaging in animal tissues.

Caspases are cysteine proteases that play key roles at different stages of programmed cell death (i.e., apoptosis) (1). Caspases are inactive in normal cells but can be activated by proapoptotic stimuli. Caspase-3 is a central effector caspase, which becomes active at relatively late stages and unites both the extrinsic and intrinsic pathways of apoptosis $(2,3)$.

The great importance of caspase- 3 stimulated researchers to develop various sensors for its activity. In particular, several genetically encoded sensors that are used to monitor the activation of caspase-3 in live cells at the single-cell level were developed.
The typical design of such sensors is based on Förster resonance energy transfer (FRET) between two appropriate GFP-like fluorescent proteins connected by a linker with the caspase-3 recognition site DEVD (4). Efficient FRET observed in a native sensor state is eliminated by linker cleavage upon caspase-3 activation. This FRET decrease can be easily monitored by standard means of FRET detection; for instance, by an increase in donor fluorescence intensity and lifetime. Caspase-3 FRET sensors were constructed using pairs of fluorescent proteins of various colors: blue and green $(5,6)$, cyan and yellow (5), yellow and red (7), or green and red (8). A drawback of such sensors is that they complicate multiparameter imaging because they occupy a significant part of the visible spectrum. In particular, a widely available collection of GFP-tagged proteins and GFP-based sensors cannot be used together with these caspase-3 sensors because of unavoidable crosstalk in the green channel. Very recently, this drawback was partially addressed by a new caspase-3 sensor named RACS3 (9). This sensor is based on FRET between the large Stokes-shifted red fluorescent protein dKeima (excitation and emission

\section{METHOD SUMMARY}

We developed a new sensor, mKate2-DEVD-iRFP, for caspase-3 activity based on FRET between the far-red fluorescent protein mKate2, which is used as the donor, and infrared fluorescent protein iRFP, which is used as the acceptor. The strongly red-shifted spectrum of the new sensor is advantageous for multiparameter imaging, as exemplified by simultaneous imaging of mKate2-DEVD-iRFP and EGFP-Bax during apoptosis. 
maxima at 440 and $570 \mathrm{~nm}$, respectively) and the mRFP1 variant FP615 (excitation and emission maxima at 590 and $615 \mathrm{~nm}$, respectively). The red-shifted emission spectrum of RACS3 allowed dual FRET imaging using RACS3 and the calcium sensor YC3.60 (which consists of the cyan ECFP and yellow Venus fluorescent proteins).

Sensors possessing fluorescence as far red-shifted as possible, however, are highly desirable for advanced multiparameter imaging, as well as for whole-body imaging in animal models, due to the nearinfrared window of transparency of animal tissues $(10,11)$. In this respect, infrared fluorescent proteins (IFPs) represent a promising basis for the construction of strongly red-shifted fluorescent sensors. IFPs are engineered bacteriophytochromes that are infrared fluorescent (excitation and emission maxima at 660-690 and 680-720 $\mathrm{nm}$, respectively) due to covalently bound biliverdin (12,13).

In a very recent report, the fluorogenic sensor of caspase-3 activity, iCasper, was created using a circularly permuted IFP (14). It was designed in such a way that the IFP contains no biliverdin cofactor in the native (uncleaved) state and develops infrared fluorescence only after cleavage by caspase-3. However, iCasper contains split GFP as an essential part of the sensor to ensure integrity of the protein chain after cleavage as well as to help visualize cells expressing iCasper. Thus, mutiparameter imaging with additional green fluorescent tags or sensors is inapplicable for iCasper in its present version (although the split GFP can potentially be swapped for a split RFP variant).

Recently, we tested FRET between far-red GFP-like fluorescent proteins and iRFP (15). iRFP, also known as iRFP713 (16), is one of the best variants of this protein class in terms of fluorescence brightness, maturation efficiency, and stability (17). Another important advantage of iRFP is that in most experimental models it does not require addition of exogenous biliverdin (17). It should be noted that excess bilivedin, which is often used for other IFPs (12), can potentially affect cell physiology (18-21). The high extinction coefficient $\left(105,000 \mathrm{M}^{-1} \mathrm{~cm}^{-1}\right)$ of iRFP makes it a potentially efficient FRET acceptor. We constructed fusion proteins in which a donor protein (mKate2, eqFP650 or eqFP670) was connected to an acceptor (iRFP) via a 17-amino acid linker containing the DEVD caspase-3 recognition site. Recombinant proteins were purified from bacteria and tested in vitro. All three fusion proteins demonstrated an expected increase of the donor fluorescence upon cleavage with caspase-3. However, the eqFP670-iRFP pair showed poor folding efficiency and low fluorescence brightness.

In the present work, we tested mKate2DEVD-iRFP and eqFP650-DEVD-iRFP sensors for detection of caspase-3 activation in the course of apoptosis in mammalian cells.

\section{Materials and methods}

\section{Genetic constructs and cell lines}

The mKate2-DEVD-iRFP and eqFP650DEVD-iRFP sensors consisted of the indicated fluorescent proteins (mKate2 and iRFP or eqFP650 and iRFP) connected by an amino acid linker GGNSGDEVDGTSVATGS, which is identical to the one used in the pCasper3-BG plasmid (Evrogen, Moscow, Russia) as described previously (15).

The plasmids for transient mammalian expression of sensors were constructed by inserting the sensors' coding sequences (obtained by PCR) into the pTagBFP-N vector (Evrogen) by replacing the TagBFP sequence using the Agel and Notl restriction sites. Where appropriate, pmKate2 (Evrogen) and pEGFP-Bax (Clontech, Palo-Alto, CA) vectors were used for transient transfections.

HeLa and CT26 (murine colon carcinoma) cell lines were cultured in DMEM with $10 \%$ fetal calf serum (GE Healthcare Life Sciences, Logan, UT), 2 mM glutamine (PanEco, Moscow, Russia), $10 \mathrm{U} / \mathrm{mL}$ penicillin, and $10 \mathrm{mg} / \mathrm{mL}$ streptomycin (PanEco). Cell cultures were maintained at $37^{\circ} \mathrm{C}$ in a humidified $5 \%$ $\mathrm{CO}_{2}$ incubator. Transient transfection was performed using X-tremeGENE 9 (Roche Diagnostics GmbH, Penzberg, Germany) or FuGene 6 (Promega, Madison, WI) transfection reagents.

Stable CT26 cell lines were created using lentiviral transduction, essentially as described earlier (22). Briefly, the PCR fragment containing the mKate2-DEVDiRFP or eqFP650-DEVD-iRFP open reading frame was cloned into $\mathrm{Ndel}$ - and Sall-digested pRRLSIN.EF1.WPRE vector kindly provided by Didier Trono (Ecole Polytechnique Federale de Lausanne, Lausanne, Switzerland), with a modified multiple cloning site. Vector particles were generated by calcium phosphate transient transfection of HEK293T cells with the two packaging plasmids pR8.91 and pMD.G, and the transfer vector plasmid (mKate2DEVD-iRFP or eqFP650-DEVD-iRFP). To create stable cell lines, $3 \times 10^{6}$ lentiviral particles were added to $1 \times 10^{5} \mathrm{CT} 26$ cells. Cell populations with the brightest fluorescence were sorted using a MoFlo fluorescence-activated cell sorter (DakoCytomation, Glostrup, Denmark).

Apoptosis was induced with $10 \mu \mathrm{M}$ staurosporine (Enzo Life Sciences, Farmingdale, NY).

\section{Fluorescence microscopy}

A BZ9000 microscope (Keyence, Osaka, Japan) with a 120W ultra-high-pressure mercury lamp and a 2/3 inch, 1.5 million pixel monochrome CCD camera was used for wide-field fluorescence microscopy. Multiposition time-lapse imaging was performed at $37^{\circ} \mathrm{C}$ in a HEPES-buffered media using the $20 \times$ objective in far-red (excitation at 540-580 nm, dichroic mirror at $595 \mathrm{~nm}$, emission at 600-660 nm), infrared (excitation at 590-650 nm, dichroic mirror at $660 \mathrm{~nm}$, emission at 670-740 nm), and when appropriate, green (excitation at 450-490 nm, dichroic mirror at $495 \mathrm{~nm}$, emission at 510-560 nm) channels.

The response of the caspase- 3 sensors was estimated as follows. For each cell in a time-lapse series, the backgroundsubtracted signal of the donor fluorescence (mKate2 or eqFP650; far-red channel) was divided by the background-subtracted signal of the acceptor fluorescence (iRFP; infrared channel). Changes in this ratio with time (as apoptosis proceeds) were normalized to the first time point.

\section{Fluorescence lifetime imaging (FLIM)}

FLIM was performed on a laser scanning confocal microscope (LSM 710; Carl Zeiss, Jena, Germany) with a FLIM module (Becker \& Hickl GmbH., Berlin, Germany). The C-Apochromat 40x/1.2 W objective was used for all measurements. Two-photon excitation of the donor protein was performed with a Ti:Sa short-impulse femtosecond laser Chameleon Vision II (Coherent, Santa Clara, CA) at 1000 nm with a modulation frequency of $80 \mathrm{MHz}$ and a pulse duration of 140 fs. Fluorescence was detected with the 595-670 $\mathrm{nm}$ emission filter. In time-lapse FLIM microscopy, images were obtained at 
A
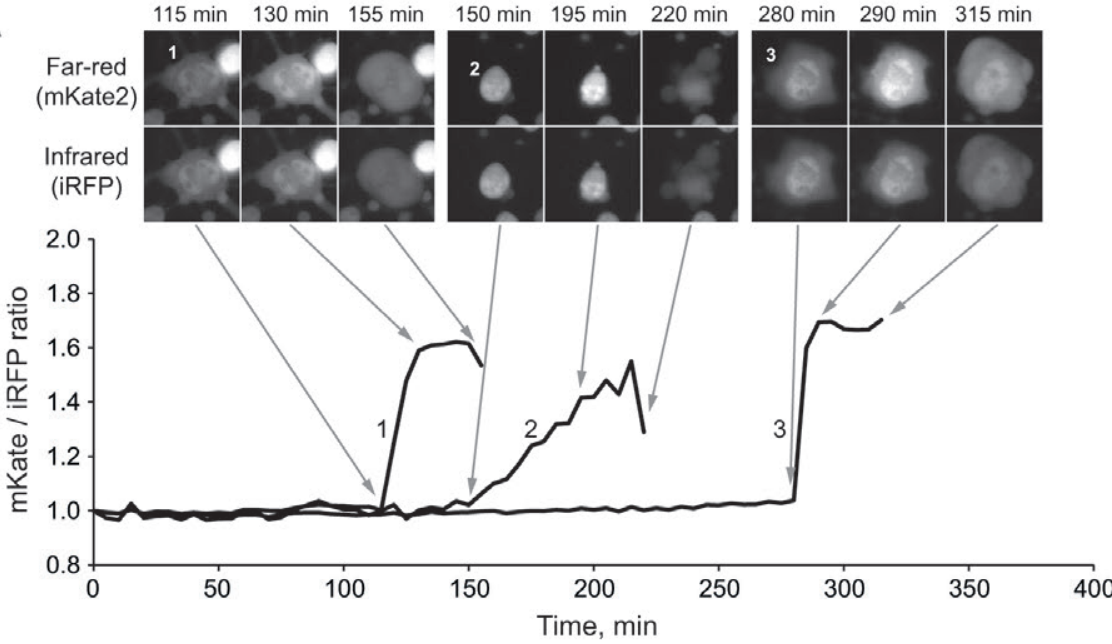

B

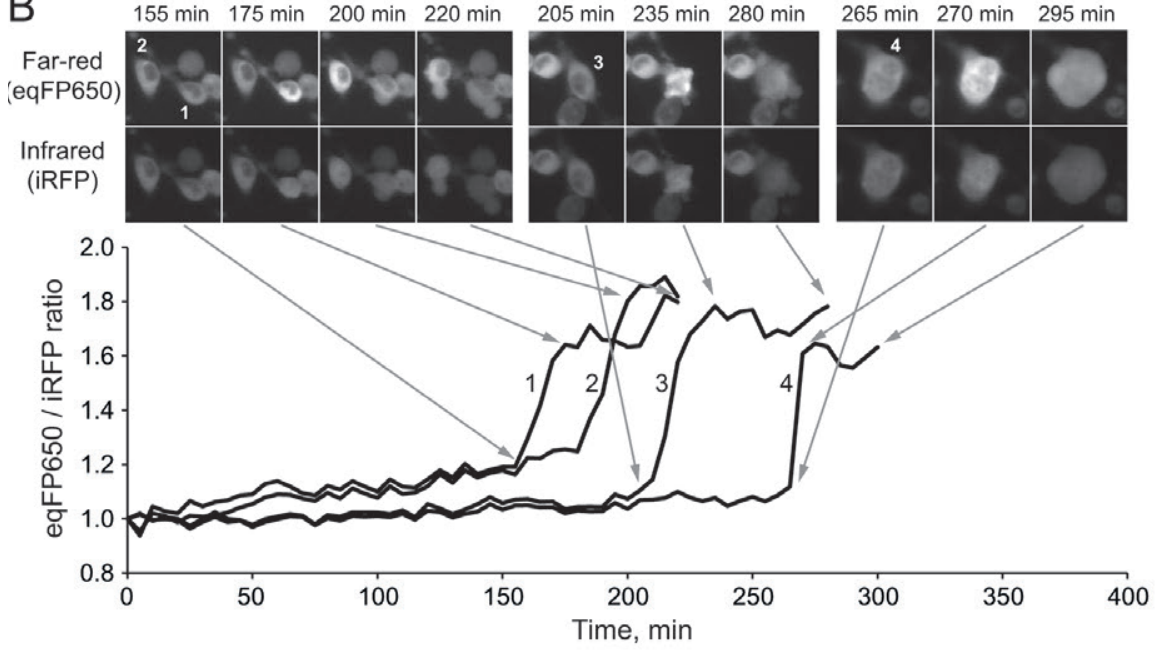

C

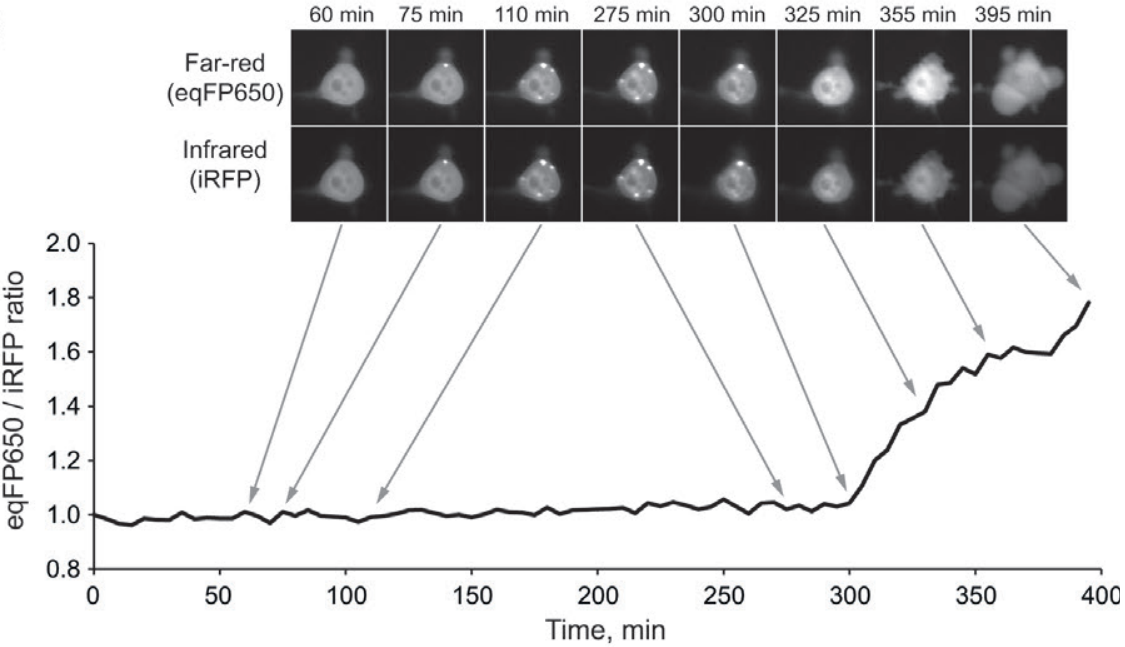

Figure 1. Response of mKate2-DEVD-iRFP and eqFP650-DEVD-iRFP sensors during staurosporineinduced apoptosis in CT26 cells. Graphs show changes in the far-red/infrared fluorescence ratio in representative cells. Cell images in the two channels at selected time points are shown. Numbers at the top indicate time in minutes after addition of staurosporine; arrows show the corresponding time point on the graphs. (A) Cells expressing mKate2-DEVD-iRFP. (B) Cells expressing eqFP650DEVD-iRFP without aggregation. (C) An example of reversible aggregation of eqFP650-DEVD-iRFP. $37^{\circ} \mathrm{C}$ and $5 \% \mathrm{CO}_{2}$ every $15-30$ min for 6 h. The FLIM data were processed using the SPCImage software (Becker \& Hickl $\mathrm{GmbH}$ ). The fluorescence lifetime of the donor alone as well as in the presence of the acceptor was measured.

\section{Results and discussion}

We constructed vectors for the expression of mKate2-DEVD-iRFP and eqFP650DEVD-iRFP in mammalian cells. After transient transfection with these vectors, HeLa cells showed evenly distributed fluorescence in the far-red and near infrared channels. Upon induction of apoptosis with staurosporine, we observed the expected increase of the donor fluorescence intensity shortly before cell shrinkage and blebbing typical for dying cells. mKate2-DEVD-iRFP and eqFP650-DEVD-iRFP demonstrated a similar (about 1.5-fold) increase of the donor signals (not shown).

For a more thorough characterization of the sensors, we generated CT26 (mouse colon carcinoma) cell lines stably expressing mKate2-DEVD-iRFP or eqFP650-DEVDiRFP. To follow apoptosis, CT26 mKate2DEVD-iRFP cells were treated with staurosporine and photographed in 2 channels (far-red and near infrared for detection of mKate2 and iRFP, respectively) every $5 \mathrm{~min}$ for several hours. Such ratiometric imaging should provide a suitable response that is insensitive to cell movement, changes of cell shape, and other events unrelated to caspase-3 activation. We observed an extended period of a constant mKate2/iRFP ratio, followed by its sharp increase (1.63 \pm 0.15 fold) (Figure 1A). A clear apoptotic change of cell morphology (shrinkage and blebbing) occurred in these cells 20-30 min after the mKate2-DEVD-iRFP sensor response. No change in the mKate2/ iRFP ratio was detected in apoptotic cells expressing a control construct in which the DEVD sequence was mutated to DEVG (data not shown). We concluded that the increase of mKate2 fluorescence intensity reflects activation of caspase- 3 and the elimination of FRET between mKate2 and iRFP, similar to the well-documented responses of other caspase-3 sensors (5-9).

Experiments on staurosporine-treated CT26 cells expressing eqFP650-DEVDiRFP demonstrated a similar behavior of this sensor with a $1.59 \pm 0.15$ fold increase of eqFP650 fluorescence intensity (Figure 1B). 
A

$5 \min$
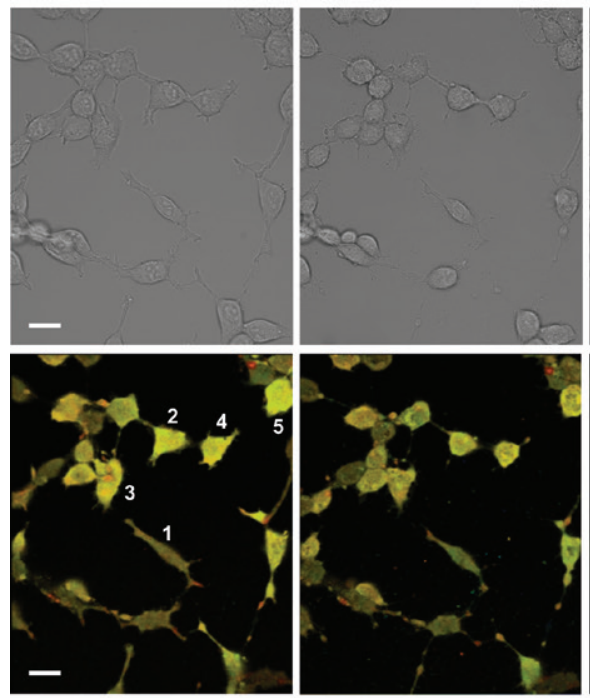

$1.0 \mathrm{~ns}$
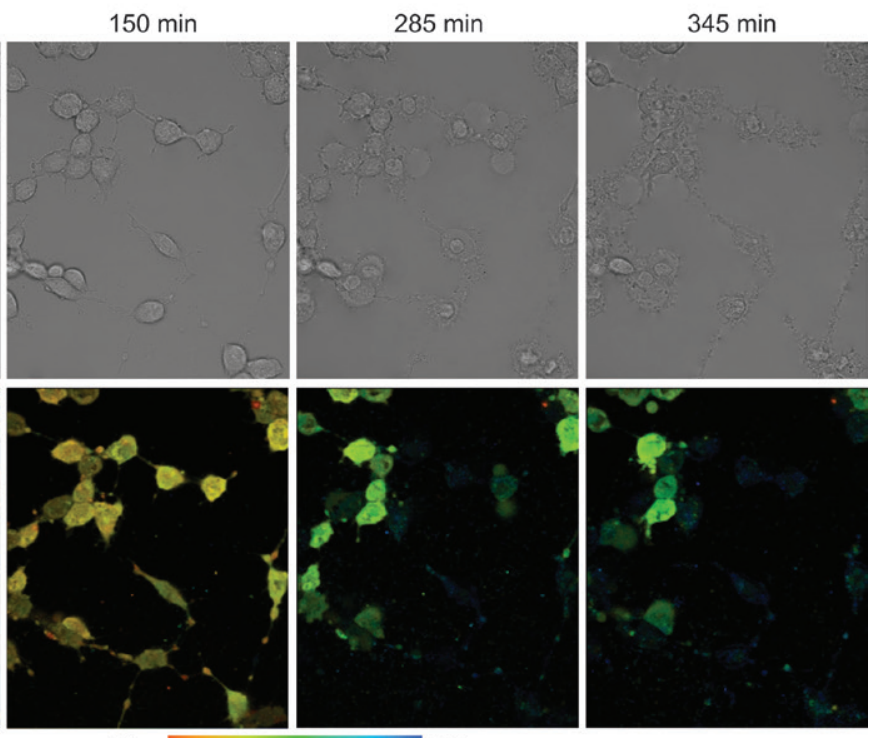

B

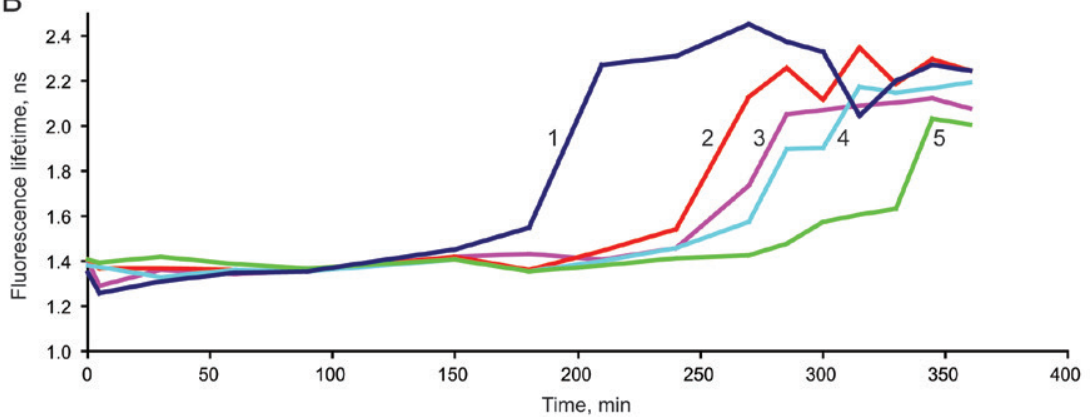

Figure 2. Fluorescence lifetime imaging (FLIM)-based analysis of mKate2-DEVD-iRFP in CT26 cells undergoing apoptosis. (A) Transmitted light (top panels) and fluorescence lifetime (bottom panels) cell images. Numbers indicate time in minutes from starosporine treatment. Scale bar: $20 \mu \mathrm{m}$. (B) Graph of changes of mean fluorescence lifetime in the cells marked in (A).

However, some cells manifested an unusual phenomenon of reversible sensor aggregation (Figure 1C). At some time points, the fluorescent signal in these cells became concentrated in several very bright dots. These nearly immobile dots persisted for a long time, but disappeared shortly before activation of the sensor response (increase of eqFP650/iRFP ratio). It is probable that aggregation of eqFP650-DEVD-iRFP occurs because of the dimeric state of both eqFP650 and iRFP. Although this aggregated sensor still responded to caspase-3 activation, this phenomenon complicates analysis. Thus, we concluded that mKate2DEVD-iRFP is the preferable far-red sensor for caspase-3 since it does not aggregate.

Notably, both sensors demonstrated a similar 1.6 -fold increase in donor fluorescence intensity upon cleavage with caspase-3 in dying cells that corresponds to $38 \%$ FRET efficiency. Considerably lower FRET efficiencies (5\% for mKate2DEVD-iRFP and 25\% for eqFP650-DEVD-
iRFP) were detected in our previous experiments with purified sensors in vitro (15). We attributed this discrepancy to incomplete maturation of the iRFP acceptor during expression in bacteria.

We next applied fluorescence lifetime imaging (FLIM) for analysis of the mKate2DEVD-iRFP sensor. Control CT26 cells expressing mKate2 alone possessed a fluorescence lifetime of $2.05 \pm 0.05$ ns, while the fluorescence lifetime of mKate2 within the mKate2-DEVD-iRFP sensor was $1.40 \pm$ $0.07 \mathrm{~ns}$, demonstrating the expected shortening of the donor fluorescence lifetime due to FRET (estimated FRET efficiency 32\%). Time-lapse FLIM microscopy of CT26 mKate2-DEVD-iRFP cells after induction of apoptosis with staurosporine allowed the detection in individual cells of an increase of mKate2 fluorescence lifetime to $2.06 \pm 0.08$ ns (Figure 2) that corresponds to the lifetime of free mKate2. Thus, FLIM microscopy provides a useful way to monitor mKate2DEVD-iRFP response due to the $45 \%$ increase of mKate2 fluorescence lifetime after sensor activation (corresponding to the FRET change from $32 \%$ to $0 \%$ ).

To demonstrate the utility of these new sensors for multiparameter imaging, we performed simultaneous monitoring of caspase-3 activity and Bax translocation during apoptosis. CT26 cells stably expressing mKate2-DEVD-iRFP were transiently transfected with EGFP-Bax. In resting cells, the green EGFP-Bax signal was evenly distributed in the cytoplasm and nuclei. The next day, apoptosis was induced by treatment with staurosporine and cells were observed for several hours in three channels: green for EGFP, far-red for mKate2, and near-infrared for iRFP. In individual cells undergoing apoptosis, we were able to clearly detect both translocation of EGFP-Bax from the cytoplasm to mitochondria (23) and an increase in mKate2 signal due to activation of caspase-3. Surprisingly, the expected order of these events-Bax translocation followed by caspase-3 activation-was observed in only a small proportion ( 6 of 65 cells; 10\%) of the cells (Figure $3 A)$. In the majority of cells ( 90\%), Bax translocation occurred 5-10 min after initiation of the mKate2 fluorescence increase (Figure 3B). Similar results were obtained in HeLa cells transiently co-transfected with mKate2-DEVD-iRFP and EGFP-Bax (data not shown).

Staurosporine is a broad-range protein kinase inhibitor (24) that is widely used as a proapoptotic stimulus, but the mechanisms of its proapoptotic action remain poorly understood (25). It is generally thought that staurosporine activates the intrinsic mitochondrial pathway of cell death, which includes Bax translocation to mitochondria, release of cytochrome $\mathrm{c}$ from mitochondria, activation of the apoptosome, and activation of caspase-9. Active caspase- 9 in turn activates downstream caspases, including caspase-3. Thus, in our experiments we expected to observe EGFP-Bax translocation first followed by an mKate2-DEVD-iRFP fluorescence change (caspase-3 activation). To our surprise, only a minor fraction of the cells showed this order of events. Conversely, the mKate2DEVD-iRFP fluorescence response mainly occurred before EGFP-Bax translocation into mitochondria. This indicates that in these cells caspase- 3 was activated in a Bax-independent manner, whereas Bax translocation was a secondary event at the late stages of apoptosis. Indeed, it 
A

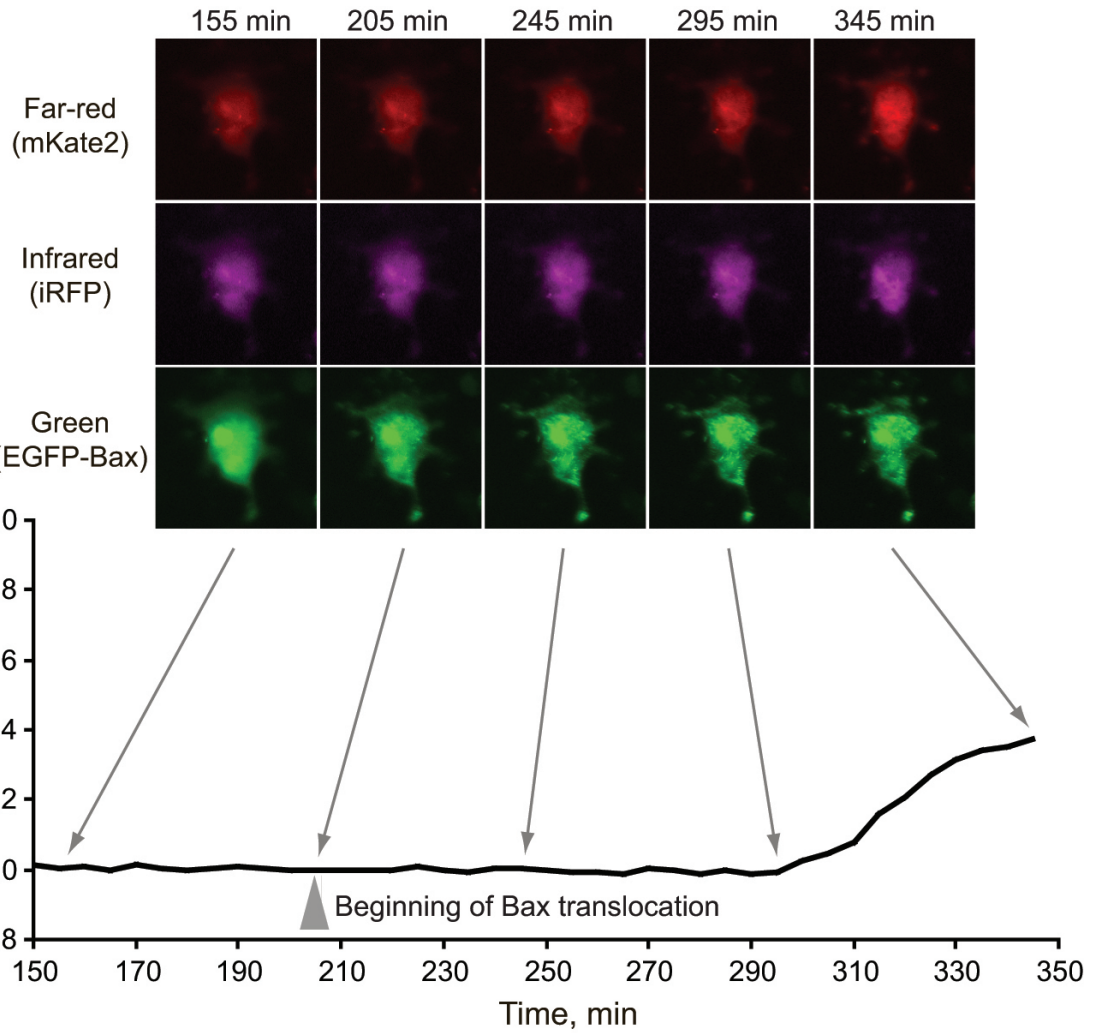

B

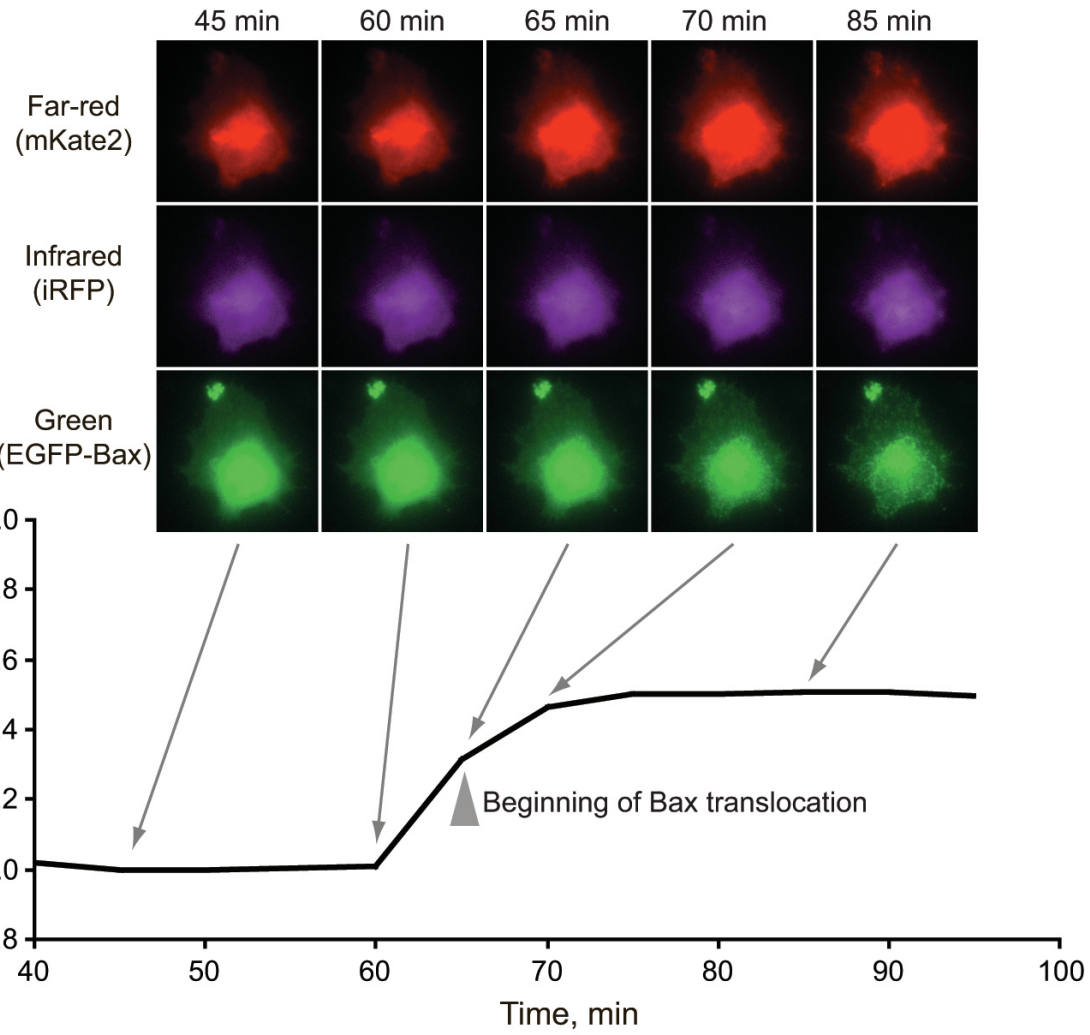

Figure 3. Simultaneous imaging of mKate2-DEVD-iRFP and EGFP-Bax during staurosporineinduced apoptosis in CT26 cells. Cells were imaged in the 3 designated channels every 5 min after treatment with staurosporine. Representative cells with early (A) and late (B) Bax translocation are shown. Time of the beginning of EGFP-Bax translocation is marked by arrowhead.

\section{IN VIVO \\ IMAGING PROBES}

Fig. Caspase/apoptosis levels in control (top) and treated (bottom) tumor-bearing mice. Imaging in live animals \& excised tumors show greater levels of apoptosis.
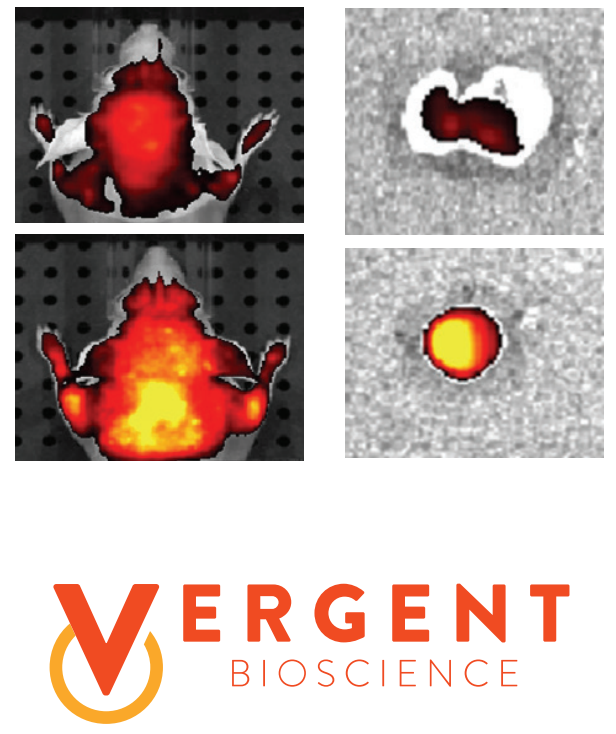

vergentbio.com/invivo 844.803.0346 
was recently demonstrated that staurosporine can directly activate caspase- 9 independently of the classical mitochondrial apoptosis pathway (25). A more detailed understanding of this non-canonical order of events that we observed during apoptosis will require further study.

In our experiments, we showed that mKate2-DEVD-iRFP is well-suited for multicolor imaging together with a green fluorescent protein. Such multiparameter imaging is very important for deciphering the true order of events and the correlations between them since individual cells behave non-synchronously. Compared to red caspase-3 sensors RACS3 (9) and TagRFP-23-KFP (26), mKate2-DEVD-iRFP possesses strongly red-shifted spectra for both donor and acceptor. Potentially, this makes it possible to use mKate2-DEVDiRFP together with orange or even red fluorescent proteins [see examples of multicolor labeling with mKate2 and mOrange2 (27) or TagRFP (28)], which are clearly incompatible with RACS3 and TagRFP-23-KFP. Also, in the case of RACS3, excitation of dKeima at $440 \mathrm{~nm}$ can result in significant cross-talk with the emission of cyan and green fluorescent proteins, which tail into the red detection channel.

Another potential advantage of the new sensor is better penetration of far-red light through animal tissues $(10,11)$. Thus, deeper imaging in animal models can be achieved compared with shorter wavelength sensors. For example, CT26 cells stably expressing mKate2-DEVD-iRFP that were generated in the present work could potentially be used to monitor apoptosis in xenograft tumors in BALB/C mice using fluorescence ratiometric or FLIM whole body imaging.

\section{Author contributions}

K.A.L., M.V.S., and E.V.Z. conceived the study. O.A.Z., T.F.S., M.V.S., V.V.D., and G.V.S. performed the experiments. K.A.L., O.A.Z., T.F.S., M.V.S., and E.V.Z. wrote the manuscript.

\section{Acknowledgments}

The authors are grateful to Vladislav Shcheslavskiy (Becker \& Hickl GmbH) for his kind assistance with FLIM. This work was supported by the Russian Foundation for Basic Research (grants 13-04-40303-H and 13-04-40304-H). The work was partially carried out using equipment provided by the IBCH Core Facility (CKP IBCH).

\section{Competing interests}

O.A.Z. and K.A.L. are authors of a patent application on the caspase-3 sensors.

\section{References}

1. Jin, Z. and W.S. EI-Deiry. 2005. Overview of cell death signaling pathways. Cancer Biol. Ther. 4:139-163.

2. Grütter, M.G. 2000. Caspases: key players in programmed cell death. Curr. Opin. Struct. Biol. 10:649-655

3. Kiechle, F.L. and X. Zhang. 2002. Apoptosis: biochemical aspects and clinical implications. Clin. Chim. Acta 326:27-45.

4. Carlson, H.J. and R.E. Campbell. 2009. Genetically encoded FRET-based biosensors for multiparameter fluorescence imaging. Curr Opin Biotechnol. 20:19-27.

5. Mahajan, N.P., D.C. Harrison-Shostak, J. Michaux, and B. Herman. 1999. Novel mutant green fluorescent protein protease substrates reveal the activation of specific caspases during apoptosis. Chem. Biol. 6:401-409.

6. Subach, O.M., I.S. Gundorov, M. Yoshimura, F.V. Subach, J. Zhang, D. Gruenwald, E.A. Souslova, D.M. Chudakov, and V.V. Verkhusha. 2008. Conversion of red fluorescent protein into a bright blue probe. Chem. Biol. 15:1116-1124.

7. Kominami, K., T. Nagai, T. Sawasaki, Y. Tsujimura, K. Yashima, Y. Sunaga, M. Tsuchimochi, J. Nishimura, et al. 2012. In vivo imaging of hierarchical spatiotemporal activation of caspase-8 during apoptosis. PLoS ONE 7:e50218.

8. Shcherbo, D., E.A. Souslova, J. Goedhart T.V. Chepurnykh, A. Gaintzeva, I.I. Shemiakina, T.W. Gadella, S. Lukyanov, and D.M. Chudakov. 2009. Practical and reliable FRET/FLIM pair of fluorescent proteins. BMC Biotechnol. 9:24

9. Miyamoto, A., H. Miyauchi, T. Kogure, A. Miyawaki, T. Michikawa, and K. Mikoshiba. 2015. Apoptosis induction-related cytosolic calcium responses revealed by the dual FRET imaging of calcium signals and caspase-3 activation in a single cell. Biochem Biophys Res Commun. 460:82-87.

10. König, K. 2000. Multiphoton microscopy in life sciences. J. Microsc. 200:83-104.

11. Deliolanis, N.C., R. Kasmieh, T. Wurdinger, B.A. Tannous, K. Shah, and V. Ntziachristos. 2008. Performance of the red-shifted fluorescent proteins in deep-tissue molecular imaging applications. J. Biomed. Opt. 13:044008.

12. Shu, X., A. Royant, M.Z. Lin, T.A. Aguilera, V. Lev-Ram, P.A. Steinbach, and R.Y. Tsien. 2009. Mammalian expression of infrared fluorescent proteins engineered from a bacterial phytochrome. Science 324:804-807.

13. Piatkevich, K.D., F.V. Subach, and V.V. Verkhusha. 2013. Engineering of bacterial phytochromes for near-infrared imaging, sensing, and light-control in mammals. Chem. Soc. Rev. 42:3441-3452.

14. To, T.-L., B.J. Piggott, K. Makhijani, D. Yu, Y.N. Jan, and X. Shu. 2015. Rationally designed fluorogenic protease reporter visualizes spatiotemporal dynamics of apoptosis in vivo. Proc. Natl. Acad. Sci. USA 112:3338-3343.
15. Zlobovskaya, O.A., K.S. Sarkisyan, and K.A. Lukyanov. 2015. Infrared fluorescent protein iRFP as an acceptor for resonance excitation energy transfer. Bioorg Khim. 41:299-304.

16. Shcherbakova, D.M. and V.V. Verkhusha. 2013. Near-infrared fluorescent proteins for multicolor in vivo imaging. Nat. Methods 10:751754.

17. Filonov, G.S., K.D. Piatkevich, L.M. Ting, J. Zhang, K. Kim, and V.V. Verkhusha. 2011. Bright and stable near-infrared fluorescent protein for in vivo imaging. Nat. Biotechnol. 29:757-761.

18. Phelan, D., G.M. Winter, W.J. Rogers, J.C. Lam, and M.S. Denison. 1998. Activation of the Ah receptor signal transduction pathway by bilirubin and biliverdin. Arch. Biochem. Biophys. 357:155-163.

19. Falchuk, K.H., J.M. Contin, T.S. Dziedzic, Z. Feng, T.C. French, G.J. Heffron, and M. Montorzi. 2002. A role for biliverdin IXalpha in dorsal axis development of Xenopus laevis embryos. Proc. Natl. Acad. Sci. USA 99:251-256.

20. Maines, M.D. 2003. Bile pigments: newcomers to the cell signaling arena. Toxicol. Sci. 71:9-10.

21. Maines, M.D. 2005. New insights into biliverdin reductase functions: linking heme metabolism to cell signaling. Physiology (Bethesda) 20:382389.

22. Ryumina, A.P., E.O. Serebrovskaya, M.V. Shirmanova, L.B. Snopova, M.M. Kuznetsova, I.V. Turchin, N.I. Ignatova, N.V. Klementieva, et al. 2013. Flavoprotein miniSOG as a genetically encoded photosensitizer for cancer cells. Biochim. Biophys. Acta 1830:5059-5067.

23. Wolter, K.G., Y.T. Hsu, C.L. Smith, A. Nechushtan, X.G. Xi, and R.J. Youle. 1997. Movement of Bax from the cytosol to mitochondria during apoptosis. J. Cell Biol. 139:1281-1292.

24. Fabian, M.A., W.H. Biggs, D.K. Treiber, C.E. Atteridge, M.D. Azimioara, M.G. Benedetti, T.A. Carter, P. Ciceri, et al. 2005. A small molecule-kinase interaction map for clinical kinase inhibitors. Nat. Biotechnol. 23:329-336.

25. Manns, J., M. Daubrawa, S. Driessen, F Paasch, N. Hoffmann, A. Loffler, K. Lauber, and A. Dieterle. 2011. Triggering of a novel intrinsic apoptosis pathway by the kinase inhibitor staurosporine: activation of caspase- 9 in the absence of Apaf-1. FASEB J. 25:32503261.

26. Rusanov, A.L., T.V. Ivashina, L.M. Vinokurov, I.I. Fiks, A.G. Orlova, I.V. Turchin, I.G. Meerovich, V.V. Zherdeva, and A.P. Savitsky. 2010. Lifetime imaging of FRET between red fluorescent proteins. J. Biophotonics. 3:774-783.

27. Cai, D., K.B. Cohen, T. Luo, J.W. Lichtman, and J.R. Sanes. 2013. Improved tools for the Brainbow toolbox. Nat. Methods 10:540-547.

28. Chudakov, D.M., M.V. Matz, S. Lukyanov, and K.A. Lukyanov. 2010. Fluorescent proteins and their applications in imaging living cells and tissues. Physiol. Rev. 90:1103-1163.

Received 21 August 2015; accepted 07 October 2015.

Address correspondence to Konstantin A. Lukyanov, Institute of Bioorganic Chemistry, Miklukho-Maklaya 16/10, 117997 Moscow, Russia. E-mail: kluk@ibch.ru

To purchase reprints of this article, contact: biotechniques@fosterprinting.comw 